\title{
Danzhi Jiangtang Capsule ameliorates kidney injury via inhibition of the JAK-STAT signaling pathway and increased antioxidant capacity in STZ-induced diabetic nephropathy rats
}

\author{
Min Sun ${ }^{1, *}$, Wenjie Bu${ }^{1}$, Yan $\mathrm{Li}^{1}$, Jianliang Zhu ${ }^{1}$, Jindong Zhao ${ }^{2}$, Pingping Zhang ${ }^{1}$, \\ Lingling Gu ${ }^{1}$, Wenna Zhang ${ }^{1}$, Zhaohui Fang ${ }^{2, *}$ \\ ${ }^{1}$ Anhui Provincial Key Laboratory of R\&D of Chinese Material Medica, School of Life Science, Anhui University, Hefei, China; \\ ${ }^{2}$ Department of Endocrinology, the First Affiliated Hospital of Anhui University of TCM, Hefei, China.
}

\begin{abstract}
Summary Danzhi Jiangtang Capsule (DJC), a traditional Chinese medicinal formula, has been used clinically in treating diabetes and diabetic nephropathy (DN). We previously demonstrated that DJC is capable of improving renal function in patients and rats with DN, but the mechanisms underlying these therapeutic benefits of DJC are not quite clear yet. In this study, STZ-induced diabetic rats were orally administered DJC for 8 weeks. Fasting blood glucose, renal function indicators in the serum, renal index, and the expression of proteins related to JAK-STAT signaling pathway were evaluated at the end of the experiment. The kidneys were sliced for pathological histology. Antioxidant status was assessed by measuring SOD, LPO and MDA in serum. The expression levels of COX2, iNOS, SOCS and the phosphorylation status of JAK2, STAT1, and STAT3 in renal tissues were evaluated by Western blot analyses. IL-6, TNF- $\alpha$, and MCP-1 expression levels in renal tissues were determined using doubleantibody sandwich ELISA. Diabetic renal dysfunction and its associated pathologies were ameliorated by DJC treatment. DJC significantly reversed the high expression of COX2 and iNOS in renal tissues. Furthermore, DJC inhibited the JAK2-STAT1/STAT3-SOCS3 signaling pathway, resulting in decreased concentrations of IL-6, TNF- $\alpha$, and MCP-1. Moreover, the oxidant status in the kidney was substantially ameliorated by DJC treatment. In conclusion, the ability of DJC to ameliorate diabetic renal dysfunction and the associated pathologies of this disease might be due to its antioxidant capacity and suppression of the JAK2-STAT1/ STAT3 cascade.
\end{abstract}

Keywords: Danzhi Jiangtang capsule, diabetic nephropathy, JAK-STAT signaling pathway, oxidative stress

\section{Introduction}

Diabetic nephropathy (DN), the most common and most serious microvascular complication of diabetic

Released online in J-STAGE as advance publication December 28, 2018.

*Address correspondence to:

Dr. Min Sun, School of Life Science, Anhui University, Hefei 230601, Anhui, China.

E-mail: sunmin@ahu.edu.cn

Dr. Zhaohui Fang, Department of Endocrinology, the First Affiliated Hospital of Anhui University of TCM, Hefei 230031, Anhui, China.

E-mail: fangzh9097@126.com mellitus (DM), is a major cause of chronic renal failure or end-stage renal disease (ESRD). With the increase of the incidence of DM, the subsequent incidence of DN is increasing worldwide. Multiple mechanisms contribute to the occurrence and development of DN, such as glucose and lipid metabolic disorders, oxidative stress, accumulation of advanced glycosylation end products (AGEs) and the interaction with their receptors, and renal hemodynamic alterations. Accumulating evidence suggests that inflammation is an important pathogenic mechanism of diabetic complications (1-4).

Elevated levels of proinflammatory cytokines, chemokines, adhesion molecules and growth factors in renal tissues, serum and urine of diabetic patients are correlated with albuminuria (1-4). During inflammation, 
cytokines and growth factors exert their functions mainly through activation of the Janus tyrosine kinase (JAK)-signal transducers and activators of transcription (STAT) pathway. The activated JAK-STAT signal cascade can accelerate the proliferation of glomerular mesangial cells and matrix expansion, contributing to DN $(5,6)$. Thus the modulation of this pathway may prevent or alleviate the progression of $\mathrm{DN}(5,7)$. The JAK-STAT cascade is a relatively simple signaling pathway with only a few principal components. There are four members in the JAK family (JAK1, JAK2, JAK3, and TYK2) and seven members in the STAT family (STAT1, STAT2, STAT3, STAT4, STAT5A/B, and STAT6). The binding of ligands to their receptors triggers the JAK-STAT signal cascade $(5,7)$ and subsequently regulates the expression of target genes encoding cytokines, chemokines, adherence molecules, and inducible enzymes such as iNOS (inducible nitric oxide synthase) and COX-2 (Cyclooxygenase 2). The JAK-STAT signaling pathway is regulated by several mechanisms, among which suppressors of cytokine signaling (SOCS) family is the most important negative regulator. SOCS family, containing at least eight members (CIS; SOCS1 to SOCS7), can be induced by many cytokines and pathogenic mediators, and thereby, serves to inhibit further signal transduction in a negative feedback loop (7-9).

Danzhi Jiangtang Capsule (DJC) is a traditional Chinese medicinal formula that has been shown to have good clinical efficacy in diabetes and DN. Our previous studies demonstrated that DJC inhibits oxidative stress and suppresses elevated inflammatory factors including IL (Interleukin)-8, tumor necrosis factor (TNF)- $\alpha$, CXCL[The chemokine (C-X-C motif) ligand]-5, 9, and MCP-1 (monocyte chemotactic protein 1) in diabetic patients and rats (10-13). DJC has also been reported to inhibit NF- $\kappa \mathrm{B}$ and ameliorate renal inflammation in diabetic rats $(10,14)$ and to reduce the urinary albumin excretion rate (UAER) in early-stage DN patients $(10,15)$.

However, the mechanisms underlying these therapeutic benefits of DJC are not quite clear yet. Therefore, the present study was carried out to evaluate the efficacy and to explore the potential underlying mechanism of DJC on DN. In this study, STZ-induced diabetic rats were treated with DJC intragastrically for 8 weeks, the antioxidant effects of DJC were evaluated and the roles of DJC in JAK-STATs signaling and SOCS proteins expression were also explored.

\section{Materials and Methods}

\subsection{Preparation of DJC}

DJC was provided by the Department of Pharmaceutics at the First Affiliated Hospital of Anhui University of Traditional Chinese Medicine (Hefei, Anhui, China).
DJC contains 6 medicinal components at a ratio of 6:5:4:4:3:3: Radix Pseudostellariae, Radix Rehmanniae, Cortex Moutan, Rhizoma Alismatis, Semen Cuscutae Chinensis, and Leech. As mentioned in the previous study (16), Cortex Moutan was extracted using ethanol and the extract was dried under vacuum. The gruffs of Cortex Moutan and the other 4 medicinal components except for Leech, were mixed and extracted with water. After vacuum drying, the dried extract powder was obtained. The ground fine powder of Leech was mixed with the above-mentioned dry extract powder, and the resulting powder was prepared as capsules with each capsule containing $0.4 \mathrm{~g}$ of extract (equivalent to $8 \mathrm{~g}$ mixture of herbal medicine). The components of the DJC capsule were detected and quantitated by ultraperformance liquid chromatography (UPLC) (17).

\subsection{Chemicals and reagents}

Rat TNF- $\alpha$ and IL-6 ELISA kits were obtained from ExCell Bio Inc (Shanghai, China), and the MCP-1 kit was from Neobioscience (Shenzhen, Guangdong, China). Serum super oxide dismutase (SOD), lipid peroxidation (LPO) and malondialdehyde (MDA) kits were purchased from Jiancheng Bio-engineering Institute (Nanjing, China). The COX2, p-STAT1 (Tyr 701) and STAT1 polyclonal antibodies were obtained from SAB (Signalway Antibody Company, College Park, MD, USA). The monoclonal antibodies against p-STAT3 (Tyr 705) and STAT3 were supplied from Cell Signaling Technology (Beverly, MA, USA). p-JAK2 and JAK2 polyclonal antibodies were from Millipore (Billerica, MA, USA). The SOCS1 polyclonal antibody was from ImmunoWay Biotechnology (Plano, TX, USA). The SOCS3 and iNOS polyclonal antibodies were obtained from Santa Cruz (Santa Cruz, CA, USA). Rabbit polyclonal $\beta$-actin antibody and all of the secondary antibodies used for Western blotting were obtained from Abmart (Hangzhou, Zhejiang, China). An electrochemiluminescence (ECL) kit and BCA protein assay kit were supplied by Thermo Scientific Pierce (Rockford, IL, USA). STZ was from Sigma Chemical Co (St. Louis, MO, USA). All chemicals used in this study were analytical grade reagents and obtained commercially.

\subsection{Animals and Experimental procedures}

Male healthy Sprague-Dawley rats, weighing $80 \pm 10$ g (4 weeks old), were provided by the Experimental Animal Center of Anhui Medical University (Hefei, Anhui, China). After feeding with a high fat diet (containing 10\% lard, 2.5\% milk powder, 5\% glucose, $10 \%$ egg yolk, $1 \%$ cholesterol, and $71.5 \%$ standard rat feed) for 4 weeks, the rats were intraperitoneally injected with STZ in citrate buffer $(\mathrm{pH} 4.5)$ at a dose of $30 \mathrm{mg} / \mathrm{kg}$ body weight weekly for 3 weeks (18). Non- 
diabetic, normal animals fed with a normal diet were injected with vehicle and used as controls (Ctrl). The rats with fasting blood glucose (FBG) levels above 16.7 $\mathrm{mmol} / \mathrm{L}$ were considered to be diabetic animals, then the diabetic rats were weighed and randomly divided into the model (DM), low-dose DJC (DJCL, $600 \mathrm{mg} /$ $\mathrm{kg} . \mathrm{d}^{-1}$ in tap water) and high-dose DJC (DJCH, 2,000 $\mathrm{mg} / \mathrm{kg} \cdot \mathrm{d}^{-1}$ in tap water) groups, each consisting of 8 rats. The low dose of DJC (equivalent dose) was calculated from the human dose on the basis of estimated body surface area. Rats in the DJCH and DJCL groups were orally administered DJC for 8 consecutive weeks, while those in the control and DM groups were given an equal volume of vehicle. Rats were placed in a temperature and humidity controlled room, free access to high fat or standard rat feed and drinking water was allowed during the experiment. Furthermore, the rats were weighed on a weekly basis. FBG measurements were taken bi-weekly using a One Touch Glucometer (Life scan, Johnson \& Johnson, Milpitas, California, USA). After 8 weeks of treatment, all rats were sacrificed for analysis of renal tissue. This study was approved by the Committee for the Care and Use of Laboratory Animals of Anhui University, and procedures were in accordance with the Guidelines for the Welfare and Ethics of Laboratory Animals of China.

\subsection{Determination of biochemical parameters}

After 8 weeks of DJC administration, the rats were fasted overnight, weighed and sacrificed under chloral hydrate anesthesia (300 mg/kg, IP), and their blood was collected and centrifuged at $1,400 \mathrm{~g}$ for $10 \mathrm{~min}$ to obtain serum. The serum urea nitrogen (BUN), creatinine ( $\mathrm{Cr}$ ), uric acid (UA), FBG, cholesterol (CHO), triglycerides (TG), low-density lipoprotein (LDL) and high-density lipoprotein (HDL) were determined using an automatic biochemistry analyzer (Dimension RxL Max, Siemens, US).

\subsection{Detection of oxidative stress indicators}

The activities of total SOD and the concentrations of MDA and LPO in the serum were assayed using commercial test kits from Jiancheng Bio-engineering Institute (Nanjing, China) following the manufacturer's instructions.

\subsection{Histopathological assay}

The kidney samples were dissected and fixed in $10 \%$ formalin, followed by dehydration and embedding in paraffin. Then the tissues were cut into $5-\mu \mathrm{m}$-thick sections and stained with H\&E (hematoxylin and eosin) and PAS (periodic acid Schiff) and then were studied; photos were taken using a binocular Olympus DX45 microscope (Olympus Corporation, Tokyo, Japan).

\subsection{Renal cytokine determination}

After mashing and homogenizing, the renal tissues were centrifuged at $4,500 \mathrm{~g}$ for $10 \mathrm{~min}$ at $4{ }^{\circ} \mathrm{C}$, the supernatants were then collected, and the levels of IL-6, TNF- $\alpha$ and MCP-1 levels were measured using commercial enzyme-linked immunoassay (ELISA) kits according to the manufacturer's instructions. Cytokine concentrations were normalized to the amount of total protein of each sample, which was determined by BCA assay (Thermo Scientific Pierce, Rockford, IL).

\subsection{Western blotting}

Renal tissues were homogenized in ice-cold RIPA buffer (50 mM Tris pH 7.5, $150 \mathrm{mM} \mathrm{NaCl,} 2$ mM EGTA, 2 $\mathrm{mM} \mathrm{Na} 3 \mathrm{VO}_{4}, 1 \mathrm{mM}$ phenylmethane sulfonyl fluoride) containing HALT protease/phosphatase inhibitor cocktail (Sangon, Shanghai, China) and lysed for 60 min. The lysates were collected and centrifuged at $4^{\circ} \mathrm{C}$ for $20 \mathrm{~min}$ at $30,500 \mathrm{~g}$. The protein concentration of the supernatant was determined using a BCA kit (Thermo Scientific Pierce, Rockford, IL). Aliquots containing $40 \mu \mathrm{g}$ of protein were separated by electrophoresis through $10 \%$ or $12 \%$ sodium dodecyl sulfate (SDS)polyacrylamide gel electrophoresis (PAGE) gels and then transferred to nitrocellulose membranes (BioRad, Hercules, CA, USA). After blocking with 5\% nonfat milk in PBST buffer (phosphate buffer saline containing $1 \%$ Tween-20) for $1 \mathrm{~h}$, the membranes were probed with primary antibodies [anti-COX2 (1:500 dilution), anti-iNOS (1:300 dilution), anti-phosphorJAK2 (1:500 dilution), anti-JAK2 (1:500 dilution), anti-phosphor-STAT1 (1:500 dilution), STAT1 (1:500 dilution), anti-phosphor-STAT3 (1:500 dilution), STAT3 (Cell 1:500 dilution), SOCS1 (1:300 dilution), SOCS3 (1:300 dilution) and actin (1:5,000 dilution)] at $4{ }^{\circ} \mathrm{C}$ overnight. After washing with PBST, the membranes were probed with appropriate secondary antibodies for $2 \mathrm{~h}$ at room temperature and finally developed using ECL detection reagent. The antibodyantigen complexes were visualized by the FluorChem ${ }^{\mathrm{TM}}$ E System (ProteinSimple, California, US), and the relative densities of the bands were evaluated and quantified using ImageJ software (NIH, Bethesda, MD, USA).

\subsection{Statistical analysis}

Data were analyzed by GraphPad Prism 5.0 Software (GraphPad Software, San Diego, CA, USA) and are represented as means \pm SEM. Statistical significance was evaluated by the One-Way Analysis of Variance (ANOVA) followed by the Student-Newman-Keuls test. $p<0.05$ was considered to be statistically significant. 
Table 1. Effect of DJC on FBG, lipid profile, and renal function parameters in diabetic rats

\begin{tabular}{lcccc}
\hline Parameters & Ctrl & DM & DJCL & DJCH \\
\hline FBG $(\mathrm{mmol} / \mathrm{L})$ & $5.32 \pm 0.88$ & $23.26 \pm 4.29^{* * *}$ & $23.19 \pm 3.93$ & $19.82 \pm 3.85$ \\
Body weight $(\mathrm{g})$ & $310.38 \pm 45.43$ & $245.14 \pm 48.55^{*}$ & $235.50 \pm 38.44$ & $257.75 \pm 31.19$ \\
Kidney weight $(\mathrm{g})$ & $1.58 \pm 0.25$ & $2.81 \pm 0.54^{* * *}$ & $2.53 \pm 0.61$ & $2.41 \pm 0.64$ \\
Renal index $(\mathrm{KW} / \mathrm{BW})$ & $0.51 \pm 0.07$ & $1.13 \pm 0.08^{* * *}$ & $1.08 \pm 0.06$ & $0.93 \pm 0.17^{\# \#}$ \\
$\mathrm{Cr}(\mu \mathrm{mol} / \mathrm{L})$ & $38.53 \pm 5.77$ & $58.57 \pm 10.24^{* * *}$ & $48.86 \pm 6.367^{\#}$ & $44.88 \pm 6.38^{\# \#}$ \\
BUN $(\mathrm{mmol} / \mathrm{L})$ & $8.61 \pm 0.37$ & $17.48 \pm 4.15^{* * *}$ & $12.05 \pm 3.06^{\#}$ & $10.4 \pm 5.34^{\# \#}$ \\
UA $(\mu \mathrm{mol} / \mathrm{L})$ & $67.75 \pm 11.04$ & $223.71 \pm 54.81^{* * *}$ & $187.15 \pm 29.81^{* * *}$ & $157.63 \pm 33.01^{* * * \# \#}$ \\
CHO $(\mathrm{mmol} / \mathrm{L})$ & $1.24 \pm 0.21$ & $2.58 \pm 0.47^{* *}$ & $1.92 \pm 0.41^{* \#}$ & $1.72 \pm 0.27^{\# \#}$ \\
TG $(\mathrm{mmol} / \mathrm{L})$ & $0.29 \pm 0.10$ & $1.62 \pm 0.36^{* *}$ & $1.33 \pm 0.28^{* *}$ & $1.21 \pm 0.33^{* * \#}$ \\
HDL $(\mathrm{mmol} / \mathrm{L})$ & $2.12 \pm 0.25$ & $1.28 \pm 0.32^{* *}$ & $1.52 \pm 0.25^{* *}$ & $1.71 \pm 0.40^{* * \#}$ \\
LDL $(\mathrm{mmol} / \mathrm{L})$ & $0.32 \pm 0.13$ & $1.15 \pm 0.28^{* *}$ & $0.63 \pm 0.25^{* \# \#}$ & $0.46 \pm 0.19^{\#}$ \\
\hline
\end{tabular}

Data are presented as the means $\pm \operatorname{SEM}\left(n=8\right.$ in the control and DJCH groups, $n=7$ in the other groups). ${ }^{*} p<0.05,{ }^{* *} p<0.01,{ }^{* * *} p<0.001, v s$. ctrl; ${ }^{\#} p<0.05,{ }^{\# \# p}<0.01,{ }^{\# \#} p<0.001, v s$. DM group.

\section{Results}

3.1. Effects of DJC on the metabolic indexes and renal function in diabetic rats induced by STZ

During the experiment, one rat in the diabetic (DM) group and one in the DJCL group died of undetermined causes. As displayed in Table 1, after 8 weeks of DJC administration, body weights of rats in the DM group were lower than those in control group $(p<0.05)$. Even at the high dose of 2,000 mg $/ \mathrm{kg}^{-\mathrm{d}^{-1}}$, DJC did not markedly influence the body weight of diabetic rats. As expected, FBG was obviously higher in the STZinduced diabetic rats than those in control group $(p<$ 0.001). DJC treatment slightly reduced FBG levels, but without significant differences. Additionally, the serum levels of CHO, TG, and LDL were markedly increased, while serum HDL was markedly reduced in diabetic rats compared with the control group (Table 1). DJCL changed serum levels of $\mathrm{CHO}$ and $\mathrm{LDL}$, and DJCH significantly changed $\mathrm{CHO}, \mathrm{TG}, \mathrm{LDL}$, and $\mathrm{HDL}$ in diabetic rats during the course of the experiment.

The renal functional alterations in the diabetic rats were evaluated by examining serum BUN, $\mathrm{Cr}$, and $\mathrm{UA}$. All these serum parameters in the diabetic group were significantly elevated $(p<0.001)$ compared with the non-diabetic group, while the parameters were obviously reduced $(p<0.01)$ after 8 weeks of DJC treatment. The kidney weight and renal index [kidney weight/body weight ratio $(\mathrm{KW} / \mathrm{BW})]$ in the diabetic rats substantially increased compared with the non-diabetic rats $(p<$ $0.001)$. The rats in the DJC treatment groups showed a significant reduction in the renal index $(p<0.01)$, but no significant reduction in body weight. These results indicate that the administration of DJC is beneficial in ameliorating $\mathrm{DN}$ in rats.

\subsection{Effect of DJC on antioxidant status in diabetic rats}

Compared with the non-diabetic rats, serum LPO and MDA levels were markedly increased $(p<0.01$ and $p$
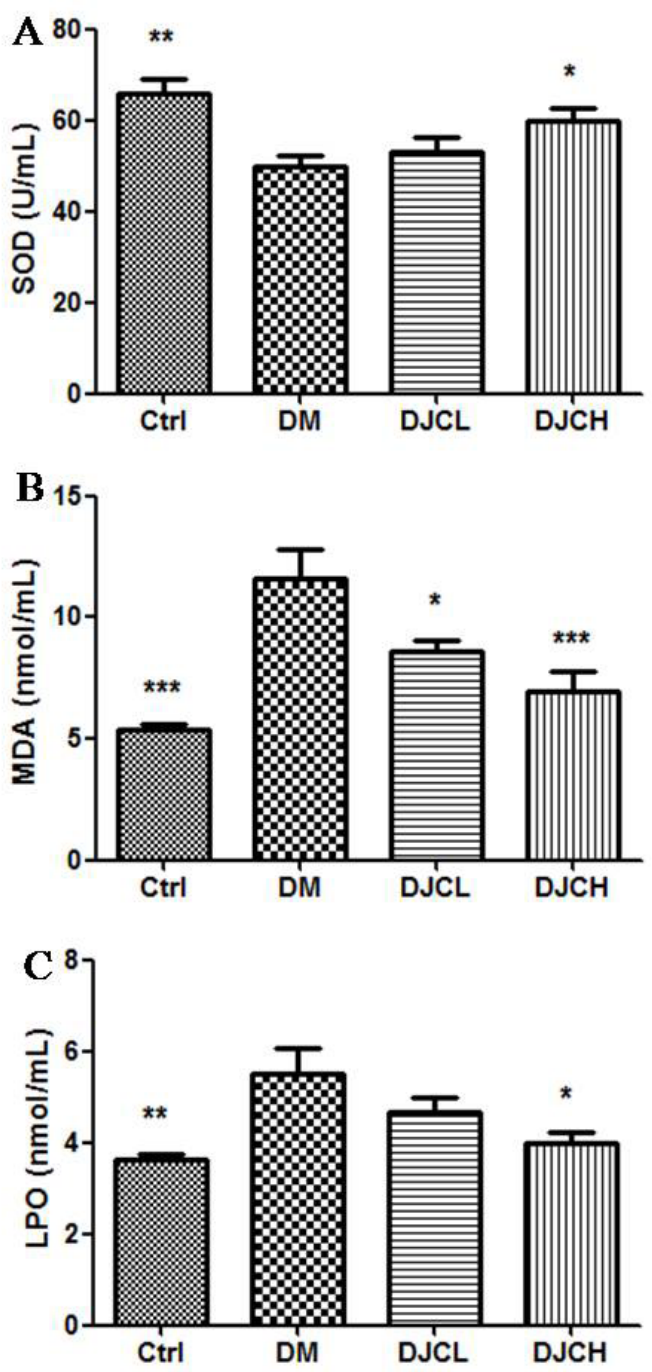

Figure 1. Effect of DJC on oxidative stress in diabetic rats. The activities of total SOD (A) and the concentrations of MDA (B) and LPO (C) in the serum were assayed using commercial test kits. Data are presented as the means \pm SEM. ${ }^{*} p<0.05$, ${ }^{* *} p<0.01,{ }^{* * *} p<0.001, v s$. DM group.

$<0.001$ ), and SOD activity was significantly decreased in the diabetic rats $(p<0.01)$, indicating that oxidative stress is initiated in diabetic rats (Figure 1). DJC markedly enhanced SOD activity $(p<0.05)$, and notably 

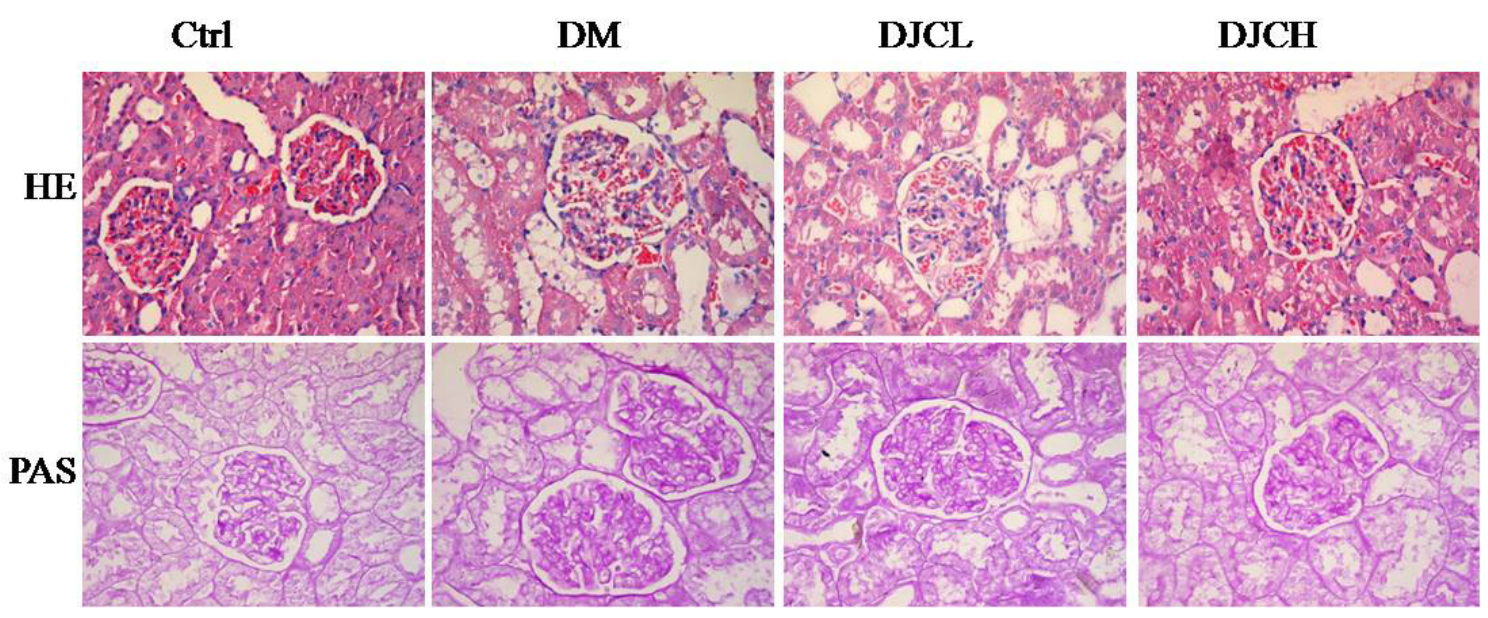

Figure 2. H\&E and PAS staining of representative sections from kidney $(\times 400)$.
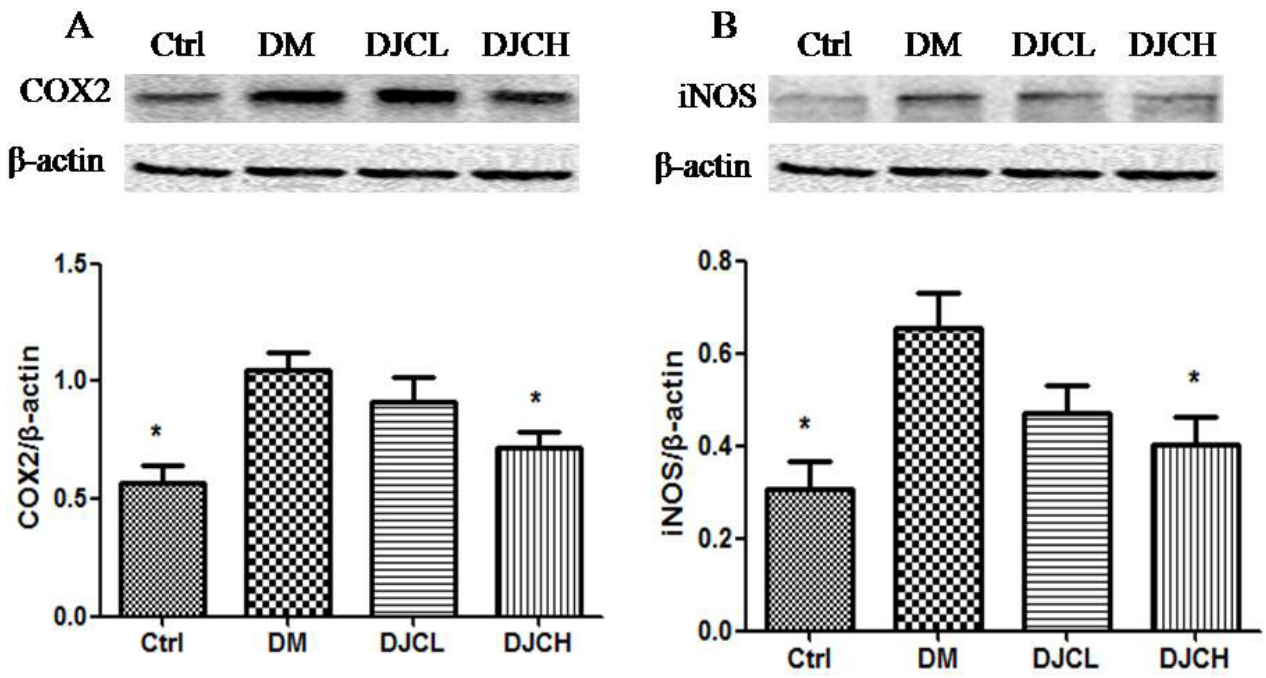

Figure 3. Effects of DJC on COX2 and iNOS expression in the diabetic rat kidneys. Total protein was extracted from the renal tissues for conducting Western blot analysis. Protein expression levels of COX2 and iNOS were normalized to the level of $\beta$-actin. (A) Quantitative analysis of COX2 expression; (B) Quantitative analysis of iNOS expression. The data are presented as the means $\pm \operatorname{SEM}, n=3 .{ }^{*} p<0.05,{ }^{* *} p<0.01,{ }^{* * *} p<0.001, v s$. DM group.

decreased LPO and MDA $(p<0.05, p<0.001)$. The results suggest that DJC is able to improve antioxidant capacity and attenuate oxidative damage in rats with DN.

\subsection{Effects of DJC on renal morphology in diabetic rats}

The representative histology of the kidney by H\&E staining is shown in Figure 2. No obvious histopathological alternations were observed in the glomerulus, kidney tubules, or mesenchyme in the renal biopsies from the animals in the control group. However, mesangial expansion with glomerular hypertrophy, basement membrane (GBM) thickening, and tubular atrophy/dilation were clearly observed in the diabetic group. The histopathological changes were clearly attenuated after DJC treatment in rats with DN. Moreover, the DJCH group showed more improvement than the DJCL group. In PAS-stained sections of the glomeruli, the mesangial matrix fraction was notably increased in the diabetic rats compared with the nondiabetic rats. This increase in the mesangial matrix fraction of diabetic rats was suppressed by DJC treatment for 8 weeks but remained higher than the control group. Taken together, these results confirmed that DJC improved renal dysfunction in diabetic rats induced by STZ.

\section{4. Effects of DJC on COX2 and iNOS expression levels in diabetic rat kidneys}

To explore the anti-inflammatory effects of DJC and its mechanism of action in the kidney, we evaluated its effect on the expression of iNOS and COX-2, key enzymes in the inflammatory processes of DN. As illustrated in Figure 3, the expression of COX2 and iNOS in the kidneys of diabetic rat were significantly increased 

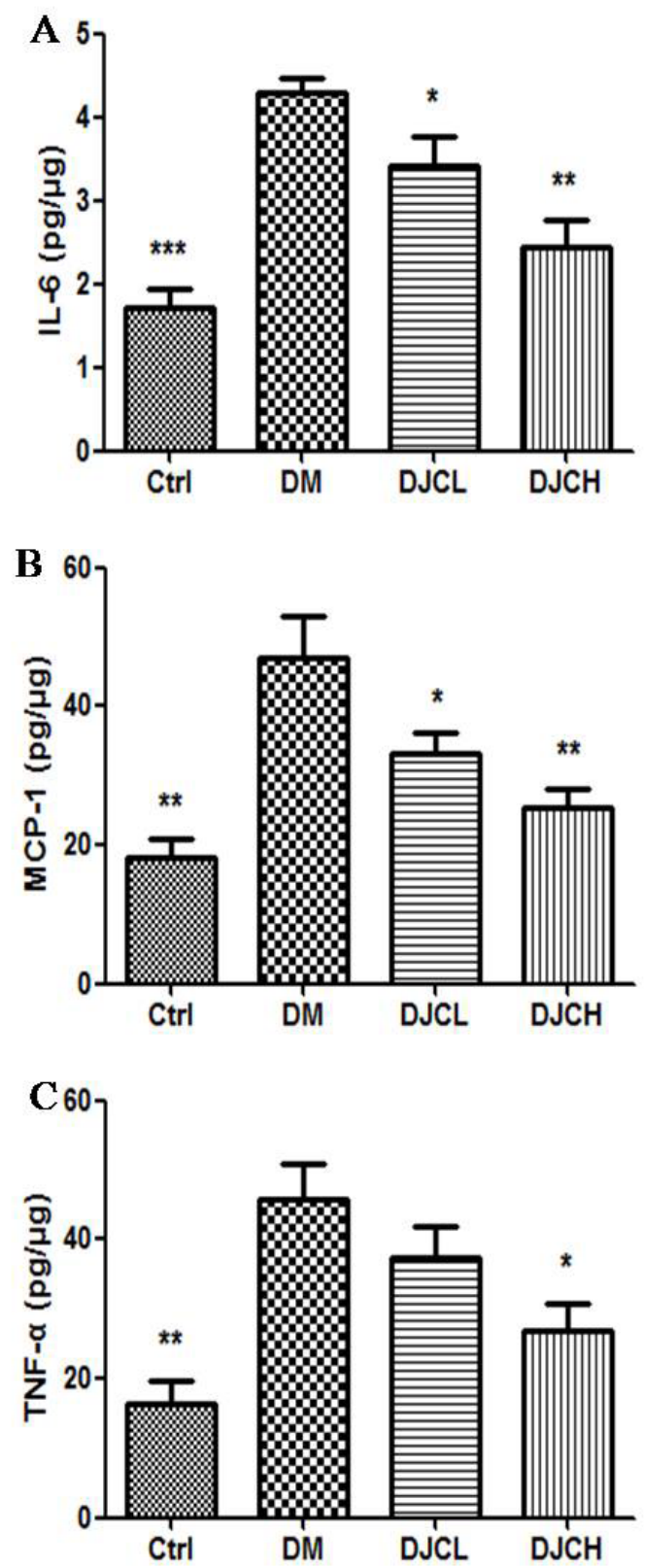

Figure 4. Effects of DJC on the inflammatory cytokine levels in diabetic rat kidneys. The renal tissues were homogenized and the supernatants were collected for cytokine detection. The levels of IL-6 (A), MCP-1 (B) and TNF- $\alpha(\mathbf{C})$ levels were measured using ELISA kits. Cytokine concentrations were normalized to the amount of total protein of each sample. Data are presented as the means $\pm \operatorname{SEM}(n=8$ in the control and DJCH groups, $n=7$ in the other groups). ${ }^{* *} p$ $<0.05,{ }^{* *} p<0.01,{ }^{* * *} p<0.001, v s$. DM group.

compared to the normal control group $(p<0.05)$. DJCH administration significantly attenuated the elevated expression of COX2 and iNOS $(p<0.05)$.

\section{5. Effects of DJC on the inflammatory cytokine levels in diabetic rat kidneys}

Inflammatory cytokines are thought to play pivotal roles in the pathogenesis of DN. Cytokines including IL-6, TNF- $\alpha$ and MCP-1 are correlated with deterioration of renal function (1-4). Therefore, to validate the effect of DJC on the JAK-STAT signaling pathway, we measured the expression profiles of IL-6, MCP-1, and TNF- $\alpha$, which contain STAT binding sites in their promoter regions. As shown in Figure 4, IL-6, MCP-1, and TNF- $\alpha$ expression levels in the diabetic group were significantly increased as compared with the non-diabetic group $(p<$ 0.01 ), administration of DJC remarkably reduced these increased inflammatory cytokines (Figure $4, p<0.05$ ).

\section{6. DJC ameliorates JAK-STAT signaling in diabetic rat kidneys}

JAK2, STAT1, and STAT3 are important signaling factors for the production of iNOS, COX2 and proinflammatory cytokines in the $\mathrm{DN}$ rat (5-7). Thus, to determine whether the inhibitory effect of DJC on the expression of proinflammatory cytokines and mediators is associated with inhibition of JAK-STAT signal pathway, we next examined the effect of DJC on the phosphorylation of JAK2, STAT1, and STAT3 in diabetic rat kidneys. The results demonstrated that the expression levels of tyrosine- phosphorylated JAK2, STAT1, and STAT3 were substantially increased in the diabetic group in comparison with the control group. DJC treatment significantly decreased the tyrosine-phosphorylated JAK2, STAT1, and STAT3 $(p<0.05$, Figure 5A, 5B, 5C), implying that the antiinflammatory activity of DJC is due to inhibition of the JAK-STAT pathway in diabetic rat kidneys.

To validate the mechanism of the JAK-STAT signaling cascade in DJC treatment, we measured the effects of DJC on SOCS1 and SOCS3 protein expression levels in DM renal tissues. As illustrated in Figure 5, the expression levels of SOCS1 and SOCS3 were significantly increased in diabetic rats compared with normal rats (Figure 5D, 5E). DJC markedly reduced the expression level of SOCS3 $(p<0.01)$, but no significant alteration was seen in SOCS1.

\section{Discussion}

In this study, intraperitoneal injections of STZ resulted in overt hyperglycemia. Kidney weight and renal index were significantly increased, and renal function (Cr, BUN, UA) was compromised. Consistently, mesangial cell proliferation, extracellular matrix (ECM) accumulation, mesangial expansion, glomerular hypertrophy, and thickened tubular and GBM were observed on the renal histopathological pictures of diabetic rats. These results indicate that the rat model of DN was successfully established. DJC treatment resulted in an obvious increase in renal function and improved associated pathologies, a decrease in renal index, and a slight reduction in blood glucose, while the general condition of the animals improved.

Growing evidence demonstrates that chronic 
$\mathbf{A}$
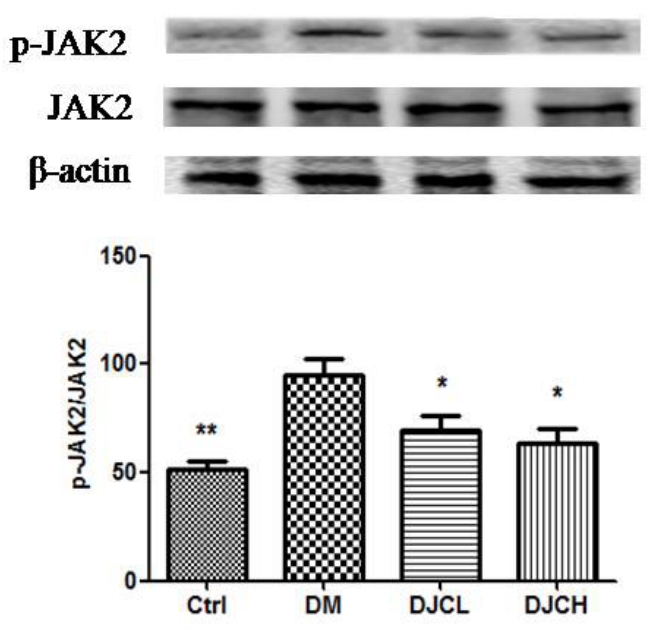

B
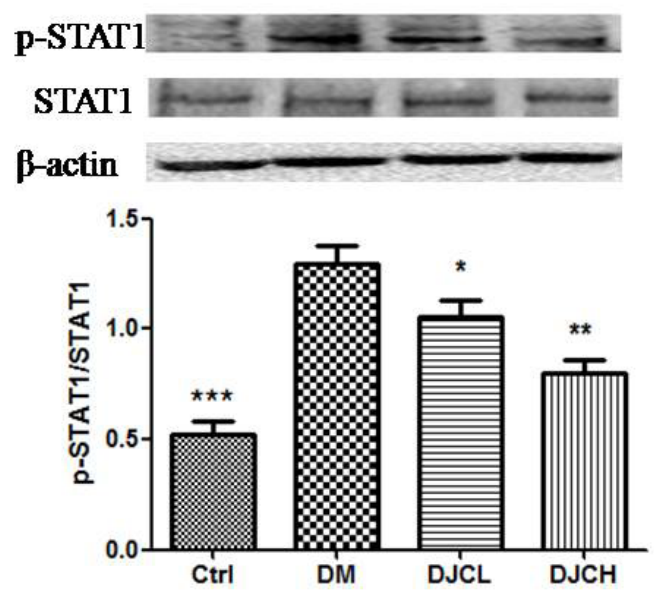

C

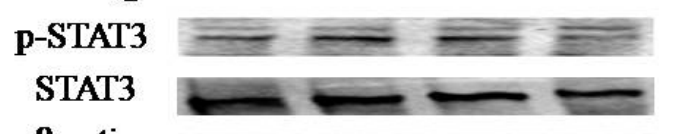

B-actin

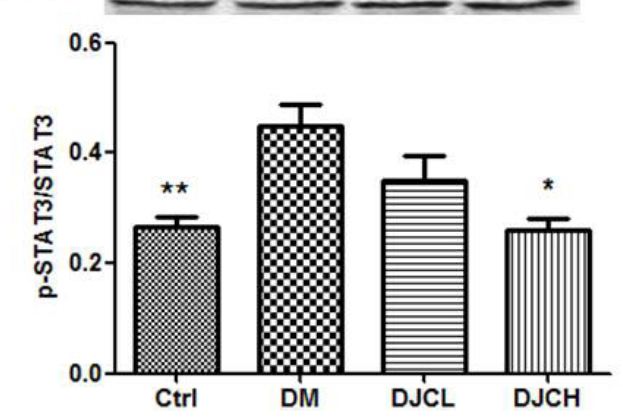

D
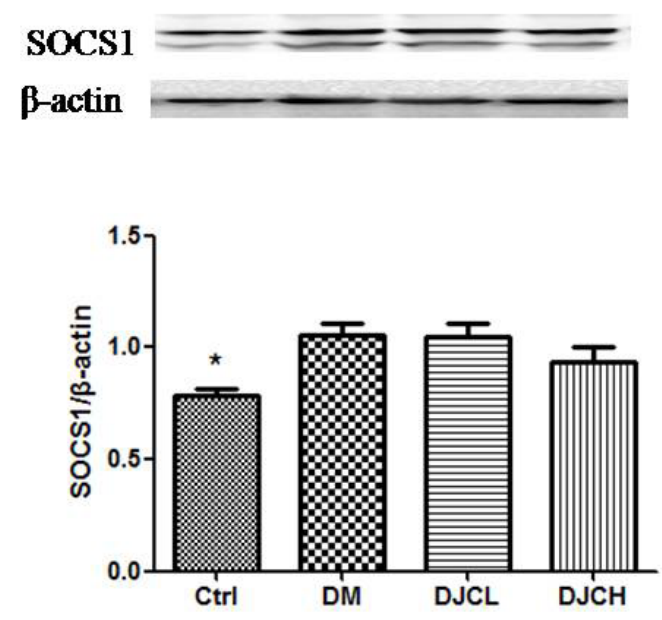

$\mathbf{E}$
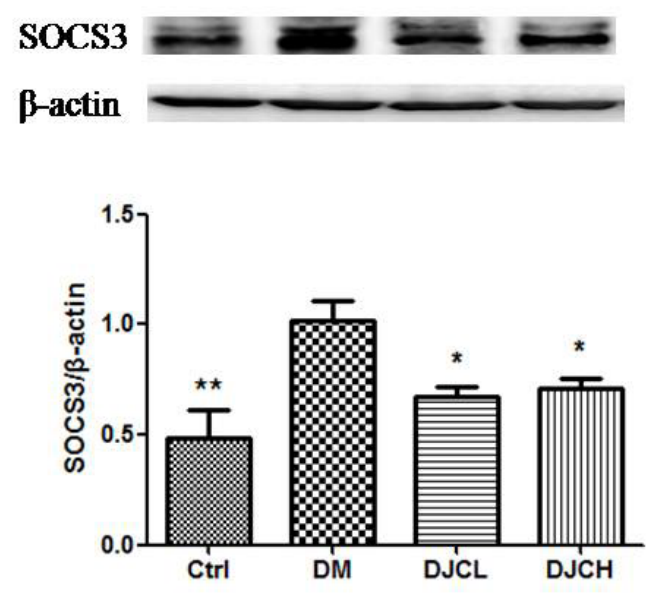

Figure 5. DJC suppressed JAK/STAT signal pathway in renal tissues of diabetic rats. Total proteins extracted from kidneys were subjected to Western blot analysis. Densitometry analysis of (A) p-JAK2/JAK2, (B) p-STAT1/STAT1, (C) p-STAT3/STAT3 expression, laser densitometry of the bands was shown in upper panels and this experiment was repeated three times. Densitometry analysis of (D) SOCS1 and (E) SOCS2 expression. Levels of SOCS proteins were normalized to $\beta$-actin. The columns and error bars represent the mean and SEM ( $n=3$ per group). ${ }^{*} p<0.05, * * p<0.01, *^{*} * p<0.001, v s$. DM.

low-grade inflammation resulting from activation of the innate immune system plays a vital role in the pathogenesis of DN (1-4). Cytokines, produced by various resident renal cells including monocytemacrophages, mesangial cells, tubular epithelial cells, podocytes, and endothelial cells, promote glomerular 
hypertrophy, glomerular cell proliferation, ECM accumulation and increase glomerular filtration rate (GFR) in early DN $(2,3)$. The glomerular injury eventually leads to albuminuria and renal failure. Circulating levels of cytokines (e.g. IL-6, MCP-1, TNF- $\alpha$ and its receptors TNFR-1 and 2) have been found correlated with albuminuria and renal function decline in DN patients (19). IL-6 has been demonstrated to play an important role in stimulating mesangial cell proliferation, promoting ECM accumulation, and increasing endothelial cell permeability $(1,2,20$ 22). A significant correlation between IL-6 and ECM accumulation, GBM thickening, and glomerulosclerosis was observed in type 2 diabetic patients with overt nephropathy (22). TNF- $\alpha$ promotes the recruitment of monocytes and macrophages, stimulates local generation of reactive oxygen species (ROS), reduces GFR by hemodynamic changes (23-25), alters endothelial permeability $(2,3,25)$, and thereby increases albumin permeability. A variety of chemokines including MCP1 can be induced by proinflammatory cytokines such as TNF- $\alpha$, IL- 6 , and interferon- $\alpha$ (INF- $\alpha)(2,26)$. MCP-1 facilitates the activation of macrophages and monocytes and their migration to lesions, and induces the expression of adhesion molecules and other proinflammatory cytokines $(3,26,27)$. MCP-1 has also been demonstrated to relate to the progression of glomerular lesions and deterioration of GFR. In the present study, DJC significantly suppressed the production of inflammatory cytokines including IL-6, TNF- $\alpha$, and MCP-1, whose promoter regions contain a potential STAT binding site. Therefore, we subsequently detected whether the activation of the JAK-STAT signaling pathway is involved in the anti-inflammatory actions of DJC in diabetic renal tissues.

JAK2-STATs signaling is activated in many cell types exposed to high concentrations of glucose and the renal cortex in rodent models of early DN $(6,7,28)$. Upon ligand binding, JAKs become activated, then phosphorylate and activate STATs. The activated STATs form dimers and translocate to the nucleus, bind to specific response elements in the promoters of target genes, and regulate the expression of target genes encoding cytokines, chemokines, adherence molecules, and inducible enzymes such as iNOS and COX2. JAK2 and its downstream signaling components, STAT1 and STAT3 are required for the production of iNOS, COX2 and proinflammatory cytokines in diabetic rat kidneys $(6,7)$. The STAT3 inhibitor, S3I201 , exhibits an inhibitory effect on renal interstitial fibroblast activation and interstitial fibrosis (29). In this study, we found that DJC treatment markedly reversed JAK2-STAT1/STAT3 activation. These results agree with previous observations $(30,31)$ and support our hypothesis that DJC ameliorates STZ-induced renal inflammatory responses via suppressing the JAK-STAT signaling pathway.
SOCS is a critical negative regulator of JAK-STAT signaling. Increased expression levels of SOCS1 and SOCS3 were detected in the STZ-induced diabetic rat model, biopsies of patients with DN and renal cells cultured with high glucose (32-34). SOCS proteins, which are rapidly induced by cytokines, have been demonstrated to regulate JAK-STAT signaling through a classic negative feedback loop (8,9). Lu et al. (35) reported that Stat3SA/- animals (transgenic mice that have a reduced capacity for STAT3 activation) with STZ-induced diabetes presented with less proteinuria, decreased mesangial cell proliferation and mesangial expansion in glomeruli, reduced macrophage infiltration, matrix accumulation and inflammatory cytokine expression at the early stage of DN. In our current study, the ability of DJC to inhibit JAKSTAT signaling was verified by the observation that it reversed the elevated protein expression level of SCOS3 in renal tissues. We also reported a similar result in mesangial cells stimulated by AGEs (16). Our results indicate that the elevated SOCS proteins are involved in the activated JAK-STAT signaling in diabetic renal tissues. However, we did not find the obvious inhibition of SOCS1 by DJC in this study. Collectively, the data presented here support a novel function of DJC and put forward a possible molecular mechanism for its action.

Hyperglycemia induces ROS, resulting in oxidative stress in the kidney. Oxidative stress is thought to contribute to the occurrence and development of DN $(36,37)$. Increased ROS interacts with the lipid in cell membranes and causes subsequent cellular damage including lipid peroxidation, which, in turn, affect cellular function. MDA, one of the final products of LPO, is considered to be a biomarker of oxidative stress and is induced by excessive ROS. SOD, a major defender against superoxide, is part of the superoxide defense system and plays an extremely important role in protecting all aerobic life-systems from oxygen toxicity. Under diabetic conditions, the balance between the oxidant/ pro-oxidant and antioxidant defense systems is destroyed and manifested as enhanced LPO and MDA and depletion of the antioxidant defense system (e.g. decreased SOD), giving rise to rapid increases in intracellular ROS levels. Antioxidants have been demonstrated to attenuate renal function decline and pathological structure in diabetic rats $(37,38)$. Chronic hyperglycemia generates ROS and oxidative stress, resulting in the activation of the JAK-STAT signaling pathway $(39,40)$. Diverse stimuli, including Ang II, cytokines, AGEs, and hyperglycemia have been reported to contribute to the development of DN through a ROS involved JAK2/STATs signaling pathway $(4,39,40)$. It is possible that inhibiting oxidative stress may suppress the JAK-STAT signaling cascade $(41,42)$. Therefore, targeting oxidative stressinduced expression of inflammatory cytokines signaling via a JAK-STAT dependent mechanism may increase 
the therapeutic effect of DN. As shown in the current study, diabetic rats exhibited significant oxidative stress, administration with DJC significantly enhanced antioxidant capacity and decreased LPO and MDA content in DN rats, indicating that DJC is capable of eliminating oxidative stress and the suppression of JAK-STAT signaling via the antioxidant defense system.

In conclusion, our results demonstrate that DJC amelioration of diabetic renal injury and associated pathology might be due to its antioxidant ability and suppression of the JAK2-STAT1/STAT3 cascade.

\section{Acknowledgements}

This work was supported by the research grant from the National Natural Science Foundation of China awarded (81573944), the research project for Practice Development of National TCM Clinical Research Bases (JDZX2012001), Natural Science Foundation of Anhui Provincial Education Department (KJ2013A027) and Key Technology R.D Program of Qinghai Province (2016-SF-126).

\section{References}

1. Wada J, Makino. Inflammation and the pathogenesis of diabetic nephropathy. Clin Sci (Lond). 2013; 124:139152.

2. Navarro-González JF, Mora-Fernández C. The role of inflammatory cytokines in diabetic nephropathy. J Am Soc Nephrol. 2008; 19:433-442.

3. Wada J, Makino H. Inflammation and the pathogenesis of diabetic nephropathy. Clin Sci (Lond). 2013; 124:139152.

4. Donate-Correa J, Martín-Núñez E, Muros-de-Fuentes M, Mora-Fernández C, Navarro-González JF. Inflammatory cytokines in diabetic nephropathy. J Diabetes Res. 2015; 2015:948417

5. Brosius FC 3rd, He JC. JAK inhibition and progressive kidney disease. Curr Opin Nephrol Hypertens. 2015; 24:88-95.

6. Marrero MB, Banes-Berceli AK, Stern DM, Eaton DC. Role of the JAK/STAT signaling pathway in diabetic nephropathy. Am J Physiol Renal Physiol. 2006; 290:F762-F768.

7. Brosius FC, Tuttle KR, Kretzler M. JAK inhibition in the treatment of diabetic kidney disease. Diabetologia. 2016; 59:1624-1627.

8. Trengove MC, Ward AC. SOCS proteins in development and disease. Am J Clin Exp Immunol. 2013; 2:1-29.

9. Linossi EM, Babon JJ, Hilton DJ, Nicholson SE. Suppression of cytokine signaling: The SOCS perspective. Cytokine Growth Factor Rev. 2013; 24:241248.

10. Fang ZH, Cheng SH, Wu Q. Effect of Danzhi Jiangtang Capsule on NF- $\kappa \mathrm{B}$ and urinary albumin excretion rate in early diabetic nephropathy patients. World Science \& Technology-Modernization of TCM and Materia Medica. 2013; 15:891-895. (in Chinese)

11. Liu SS, Li ZN, Xu CQ, Xiong YY, Fang ZH. Effects of Danzhi Jiangtang Capsule on serum levels of Tumor Necrosis Factor Alpha and Chemokine (C-X-C Motif) ligand 5 in diabetic rats. Journal of Anhui University of Chinese Medicine. 2014; 33:58-61. (in Chinese)

12. Bao TT, Yang C, Chu QG. Effects of Dangzhi Jiangtang Capsules on abdominal aortic ultrastructure, MCP-1 and resistin mRNA expression in diabetic rats. Chinese Traditional Patent Medicine. 2015; 37:1888-1892. (in Chinese)

13. Li ZN, Xiong YY, Li L, Fang ZH. Effects of Danzhi Jiangtang Capsule on CXCL9 and IL-8 in diabetic model rats. Chinese Journal of Information on TCM. 2014; 21:45-47. (in Chinese)

14. Xiong YY, Li ZN, Liu SS. The influence of Danzhi Jiangtang Capsule on the expression of NF- $\mathrm{BB}$ in kidney tissue of diabetic rats. Global Journal of Endocrinology and Metabolism Studies. 2014; 34:6-10. (in Chinese)

15. Niu YF, Fang ah, Liu J, Shi GB. The clinical efficacy of Danhzi Jiangtang Capsule on elderly patients with early diabetic nephropathy. Modern Chinese Medicine. 2008; 10:36-38. (in Chinese)

16. Sun M, Li Y, Bu WJ, Zhao J, Zhu J, Gu L, Zhang P, Fang Z. DJC suppresses Advanced Glycation End ProductsInduced JAK-STAT signaling and ROS in mesangial cells. Evid Based Complement Alternat Med. 2017; 2017:2942830.

17. Wang YZ, Jiang L, Han YQ, Zuo D, Xia LZ. UPLC fingerprint of Danzhi Jiangtang Capsule. Clinical Journal of Traditional Chinese Medicine. 2012; 24:1009-1011. (in Chinese)

18. Wang HJ, Jin YX, Shen W, Neng J, Wu T, Li YJ, Fu ZW. Low dose streptozotocin (STZ) combined with high energy intake can effectively induce type 2 diabetes through altering the related gene expression. Asia Pac J Clin Nutr. 2007; 16 Suppl 1:412-417.

19. Barutta F, Bruno G, Grimaldi S, Gruden G. Inflammation in diabetic nephropathy: Moving toward clinical biomarkers and targets for treatment. Endocrine. 2015; 48:730-742.

20. Feigerlová E, Battaglia-Hsu SF. IL-6 signaling in diabetic nephropathy: From pathophysiology to therapeutic perspectives. Cytokine Growth Factor Rev. 2017; 37:57-65.

21. Navarro-González JF, Mora-Fernández C, Muros de Fuentes M, García-Pérez J. Inflammatory molecules and pathways in the pathogenesis of diabetic nephropathy. Nat Rev Nephrol. 2011; 7:327-340.

22. Horii Y, Muraguchi A, Iwano M, Matsuda T, Hirayama T, Yamada H, Fujii Y, Dohi K, Ishikawa H, Ohmoto Y. Involvement of IL-6 in mesangial proliferative glomerulonephritis. J Immunol. 1989; 143:3949-3955.

23. Omote K, Gohda T, Murakoshi M, Sasaki Y, Kazuno S, Fujimura T, Ishizaka M, Sonoda Y, Tomino Y. Role of the TNF pathway in the progression of diabetic nephropathy in KK-A(y) mice. Am J Physiol Renal Physiol. 2014; 306:F1335-F1347.

24. Awad AS, You H, Gao T, Cooper TK, Nedospasov SA, Vacher J, Wilkinson PF, Farrell FX, Brian Reeves W. Macrophage-derived tumor necrosis factor- $\alpha$ mediates diabetic renal injury. Kidney Int. 2015; 88:722-733.

25. Navarro JF, Mora-Fernández C. The role of TNF-alpha in diabetic nephropathy: Pathogenic and therapeutic implications. Cytokine Growth Factor Rev. 2006; 17:441-450.

26. Moreno JA, Moreno S, Rubio-Navarro A, Gómez- 
Guerrero C, Ortiz A, Egido J. Role of chemokines in proteinuric kidney disorders. Expert Rev Mol Med. 2014; 16:e3.

27. Awad AS, Kinsey GR, Khutsishvili K, Gao T, Bolton WK, Okusa MD. Monocyte/macrophage chemokine receptor CCR2 mediates diabetic renal injury. Am J Physiol Renal Physiol. 2011; 301:F1358-F1366.

28. Berthier CC, Zhang H, Schin M, et al. Enhanced expression of Janus kinase-signal transducer and activator of transcription pathway members in human diabetic nephropathy. Diabetes. 2009; 58:469-477.

29. Pang M, Ma L, Gong R, Tolbert E, Mao H, Ponnusamy M, Chin YE, Yan H, Dworkin LD, Zhuang S. A novel STAT3 inhibitor, S3I-201, attenuates renal interstitial fibroblast activation and interstitial fibrosis in obstructive nephropathy. Kidney Int. 2010; 78:257-268.

30. Wang X, Shaw S, Amiri F, Eaton DC, Marrero MB. Inhibition of the Jak/STAT signaling pathway prevents the high glucose-induced increase in TGF-beta and fibronectin synthesis in mesangial cells. Diabetes. 2002; 51:3505-3509.

31. Shi Y, Zhang Y, Wang C, Du C, Zhao S, Qi Z, Zhang Q, Duan H. Suppressor of cytokine signaling-1 reduces high glucose-induced TGF-beta1 and fibronectin synthesis in human mesangial cells. FEBS Lett. 2008; 582:34843488.

32. Liu Q, Xing L, Wang L, Yao F, Liu S, Hao J, Liu W, Duan H. Therapeutic effects of suppressors of cytokine signaling in diabetic nephropathy. J Histochem Cytochem. 2014; 62:119-128.

33. Zhou Y, Lv C, Wu C, Chen F, Shao Y, Wang Q. Suppressor of cytokine signaling (SOCS) 2 attenuates renal lesions in rats with diabetic nephropathy. Acta Histochem. 2014; 116:981-988.
34. Ortiz-Muñoz G, Lopez-Parra V, Lopez-Franco O, Fernandez-Vizarra P, Mallavia B, Flores C, Sanz A, Blanco J, Mezzano S, Ortiz A, Egido J, Gomez-Guerrero C. Suppressors of cytokine signaling abrogate diabetic nephropathy. J Am Soc Nephrol. 2010; 21:763-772.

35. Lu TC, Wang ZH, Feng X, Chuang PY, Fang W, Shen Y, Levy DE, Xiong H, Chen N, He JC. Knockdown of Stat3 activity in vivo prevents diabetic glomerulopathy. Kidney Int. 2009; 76:63-71.

36. Giacco F, Brownlee M. Oxidative stress and diabetic complications. Circ Res. 2010; 107:1058-1070.

37. Stanton RC. Oxidative stress and diabetic kidney disease. Curr Diab Rep. 2011; 11:330-336.

38. Nam SM, Lee MY, Koh JH, Park JH, Shin JY, Shin YG, Koh SB, Lee EY, Chung CH. Effects of NADPH oxidase inhibitor on diabetic nephropathy in OLETF rats: The role of reducing oxidative stress in its protective property. Diabetes Res Clin Pract. 2009; 83:176-182.

39. Simon AR, Rai U, Fanburg BL, Cochran BH. Activation of the JAK-STAT pathway by reactive oxygen species. Am J Physiol. 1998; 275 (6 Pt 1):C1640-C1652.

40. Duhé RJ. Redox regulation of Janus kinase: The elephant in the room. JAKSTAT. 2013; 2:e26141.

41. Schieffer B, Luchtefeld M, Braun S, Hilfiker A, HilfikerKleiner D, Drexler H. Role of NAD(P)H oxidase in angiotensin II-induced JAK/STAT signaling and cytokine induction. Circ Res. 2000; 87:1195-1201.

42. Lee HB, Yu MR, Yang Y, Jiang Z, Ha H. Reactive oxygen species-regulated signaling pathways in diabetic nephropathy. J Am Soc Nephrol. 2003; 14(8 Supp1 3):S241-S245.

(Received October 21, 2018; Revised October 28, 2018; Accepted December 12, 2018) 